\title{
EMI REDUCTION OF PCB BY USING OPTICAL WIRING METHOD
}

\author{
Michael Mahesh K., \\ ${ }^{1}$ Department of ECE, St. Joseph College of Engineering, Chennai, India
}

\begin{abstract}
This paper describes a new optical link system which consists of a metal optical bench, a module printed circuit board, a driver/receiver integrated circuit, a vertical-cavity surface-emitting laser/photo diode (VCSEL/PD) array, and an optical link block with plastic optical fibers for reducing electromagnetic interference (EMI) noise. Optical connections are made between light sources and detectors. Because of optical wiring method no EMI noise will occurs and also the assembly of the system is easy. This method can provide robust, cost-effective, and easy-repair. This method successfully achieved a $4.5 \mathrm{~GB} / \mathrm{s}$ data transmission rate without $\mathrm{EMI}$ problems.
\end{abstract}

Keywords Electromagnetic interference (EMI), integrated optoelectronics, plastic optical fibers (POFs), optical fiber connecting, optical interconnections.

\section{INTRODUCTION}

The demand for very short-length high data rate inter-connections is increasing considerably. Parallel links are widely used in telecommunication switching systems, local computer networks, server clusters and computer systems with multithreading CPU. However, as information is usually transferred by means of an electrical signal over a relatively short distance such as between two chips or between two boards, there is a limit in increasing signal transfer speed and line density. Electrical interconnections always exhibit electrical problems such as electromagnetic interference (EMI), shorting, and ground loops.

In this paper, an optical interconnection to reduce electrical problems is introduced. In general, when an optical glass fiber does not have a jacket it lacks flexibility. Also, finishing cut ends is not easy and requires a dedicated cutting machine. . Optical backplanes with embedded waveguides and flexible polymer waveguide layers can be another great alternative. In this paper, an optical wiring method and the optical transmission media to interconnect between an optical Tx and an optical Rx, e.g., plastic optical fibers (POFs) instead of multimode glass fiber which is used largely for optical parallel interconnection is proposed. The results clearly demonstrate that the use of an optical wiring method can provide robust and cost-effective assembly for reducing EMI in chip-to-chip interconnections.

\section{ARCHITECTURE OF THE OPTICAL LINK SYSTEM}

This paper provides a method of optical interconnection between an optical transmitter and an optical receiver, mentioned in the following steps: 1) forming a light source device, an optical detection device, an optical transmission unit on a substrate [metal optical bench (MOB)] 2) Preparing a flexible optical transmission-connection medium (optical wiring link) to optically connect the light source device formed on the substrate with the optical detection device 3) directly connecting one end of the surface-finished optical transmission connection medium with the light source device and the other end with the optical detection device. Fig. 1 shows the schematic diagram of the optical Chip-to-Chip interconnects system. The basic components are lossless graded-index (GI) multimode and downsized POFs. The TRx boards contain $\mathrm{Tx}$ and $\mathrm{Rx}$ modules that consist of a metal optical bench (MOB), VCSEL arrays, PD arrays, an optical link, and high-speed access lines.

The POF is cheap, flexible, easily connectable, and it has very small bending losses and no EMI problems. The emitting beams from VCSELs are coupled into POFs with a core diameter of 62.5 micro meters. Subsequently; the beams are guided through the POFs with a 180 bended shape and then focused into the aperture of PD with a diameter of 70 micro meters. 


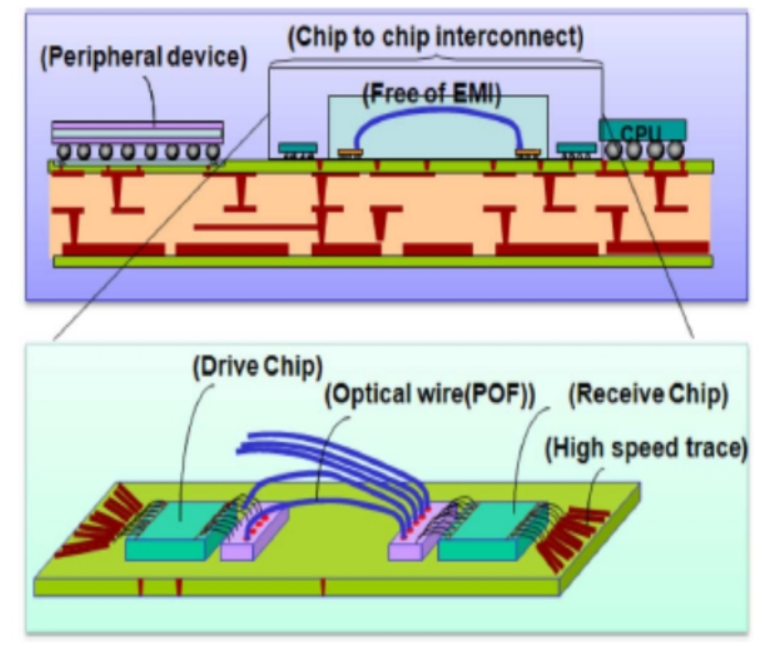

Fig 1. Architecture of an optical link system using an optical wiring link block.

\section{FABRICATION OF OPTICAL LINK SYSTEM}

\section{A. High-Speed Access Line of Optical Module PCB}

To ensure the reliable operation, differential signaling in the electrical input/output access lines is usually employed. The common mode noise is reduced by using differential signaling. To support differential signaling, differential transmission lines are required. To design the differential line differential impedance is the critical factor. The impedance affects signal qualities, such as reflections and crosstalk in both the driver and receiver. The structure of the differential lines was simulated using an ADS Momentum simulator. Finally, the width of the trace and the space within the differential pair are determined as 75 and 90 micro meters, respectively.

Time domain measurement is a useful technique for analyzing the pulse propagation within digital system interconnections. The domain reflectometry (TDR) represents the time signature of the reflected wave form. The pulse generated propagates through a coaxial cable, via the transmission line on the evaluation board, which goes through the transmitter module and reaches the input stage of the integrated circuit (IC) driver chip where it gets absorbed by transmission resistors. The differential impedance of all transmission lines and discontinuity points is about 100 plus or minus 10 ohms.

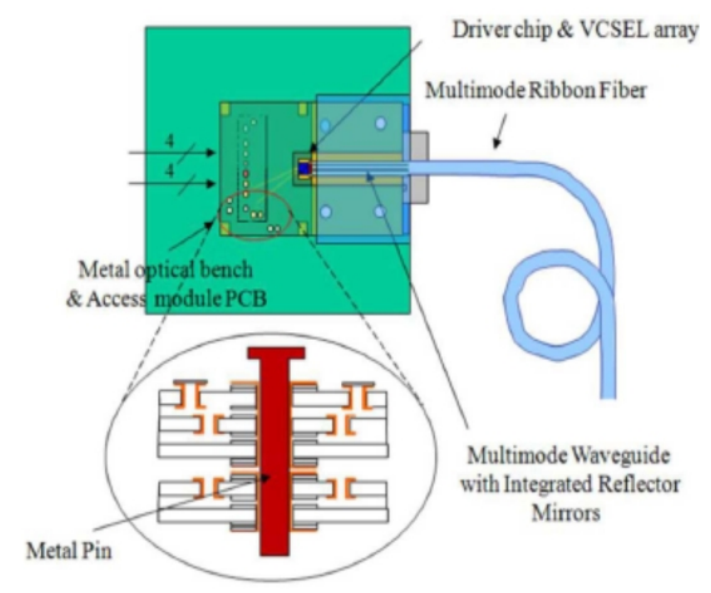

(a)

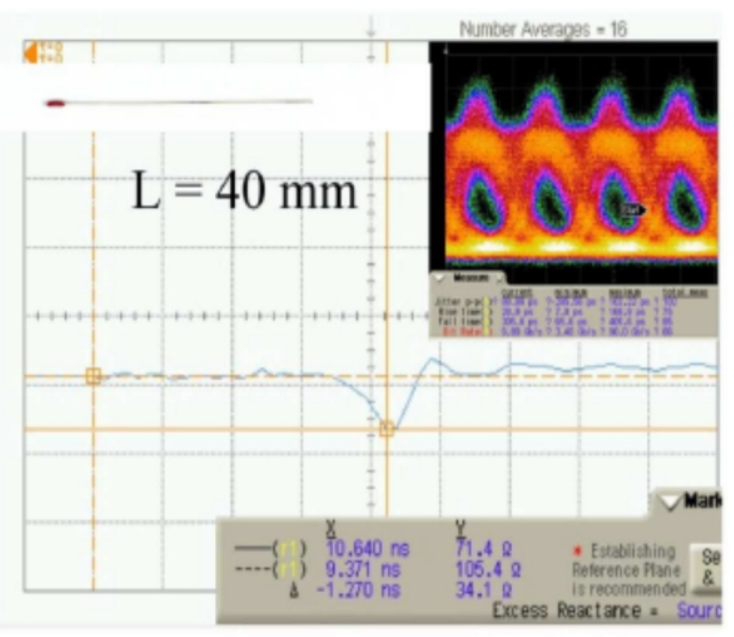

(b)

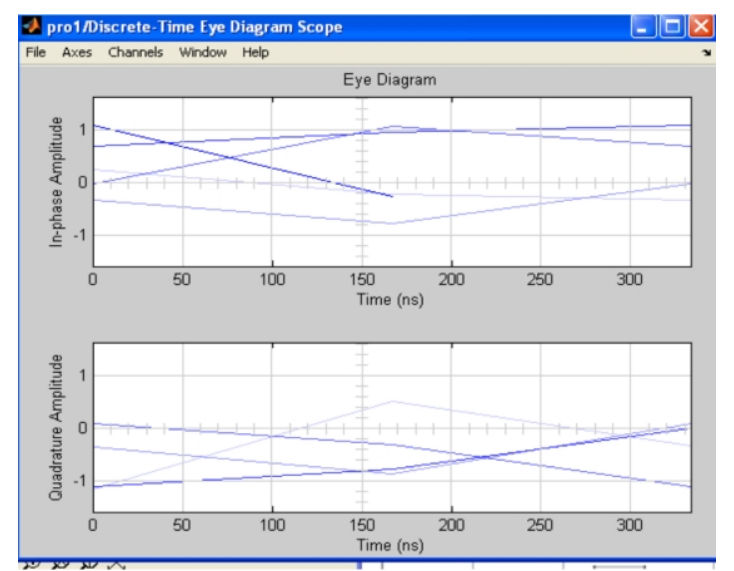

(c)

Fig 2. (a) Metal pin structure of processing board. (b) Eye diagram of Tx module with long interconnects. (c) Eye diagram of Tx module with short interconnects. 
Fig. 2 shows the metal pin structure, TDR and Eye diagram of the $T x$ module to illustrate the importance of impedance mis-matching. . 2(b) is the figure of the electrical interconnects between the module PCB and the evaluation board, which were made using long pin inter-connectors. Fig. 2(c) shows the figure of the electrical interconnects with short pin inter-connectors. The longer interconnection has more discontinuity points. It introduces the greater distortion of the eye pattern. All EMI problems can be solved by using fiber optic technology. In fact, fiber optic technology offers unique advantages in achieving a total EMl-free signal transmission within PCB modules and systems. The optical cables are immune to EMI because they transmit signals as light rather than electrical current. However, electromagnetic interference sources exist in the $E / O$ conversion parts, optical driver circuits; electrical pin interconnects, and access PCB lines.

\section{B. Optical Link Block by Using Optical Wiring Method}

An ultraviolet (UV) epoxy, a UV hardening resin, or similar binding agent may be used as the adhesive. An injector, a pipette, or similar tool may be used to precisely drop the adhesive into the aperture on the VCSEL device. Fig. 3 shows the structure of the metal optical bench

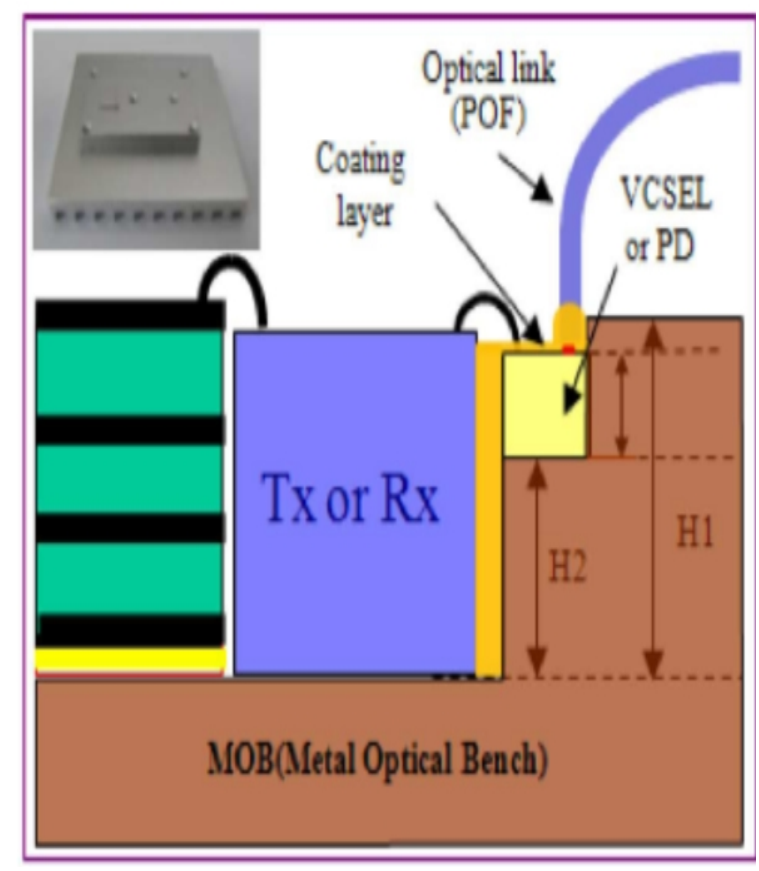

Fig 3. Structure of the metal optical bench.

\section{TRANSMISSION CHARACTERISTICS OF OPTICAL LINK SYSTEM}

\section{A. Transmission Characteristics of Optical Systemy}

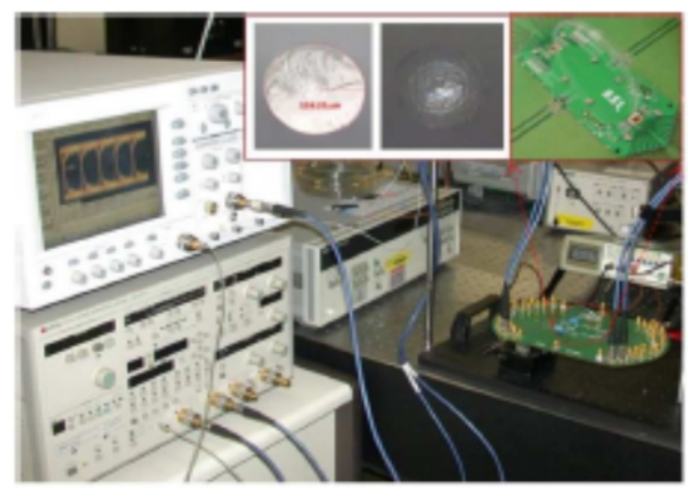

(a)

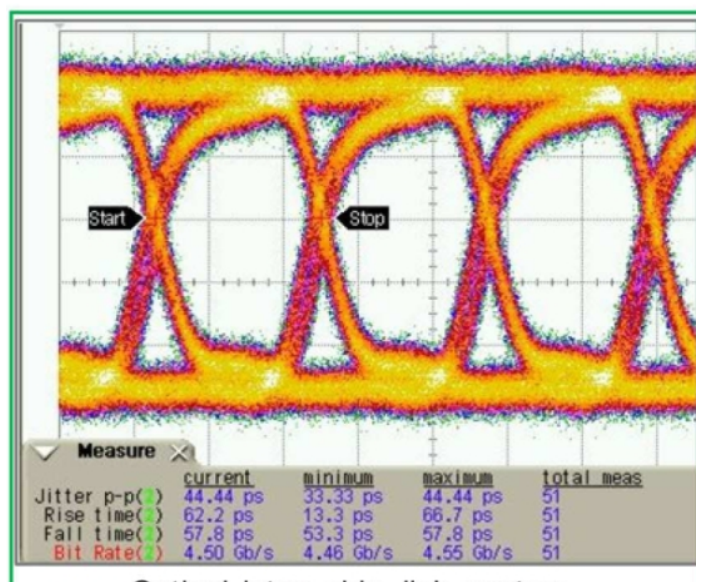

Optical inter- chip link system

(b)

Fig 4. (a) Chip-to-chip link system with an optical link. (b) Eye-diagram measured for $4.5 \mathrm{Gbp} / \mathrm{s}$ transmission of a 231-1 PRBS NRZ data

Fig. 4 shows a chip-to-chip link system with an optical link for reducing EMI. The number of channels for the optical link is 10. The eye-diagram for the transmission of $4.5 \mathrm{~Gb} / \mathrm{s}$ PRBS NRZ data is symmetric without any significant relaxation oscillation. The fgure on the upper right corner represents the enlarged photograph of a POF wiring link and the figures on the top right show the sectional picture of the cut POF and an annealed one using a razor and an iron, respectively. The channel's skew, the jitter peak to peak and the rise time of the optical link system are below 29 ps, 44.4 ps, and 62.2 ps, respectively. In this system, while the power budget of the optical link 
system was $18 \mathrm{~dB}$, total power loss of the optical link was only $5.7 \mathrm{~dB}$.

This method simplifies the fabrication process, can be easily applied regardless of various device modifications, and can reduce time taken to assemble an optical connection structure. However, further research is needed to devise adhesion materials with a high adhesive strength and a high flexible POF. Research on the smaller detector opening and the longer fiber length is required to extend data rates per channel and many applications.

\section{CONCLUSION}

An optical link system using an optical wiring method is developed. These optical link blocks based on POFs can provide a robust and cost-effective assembly for vertical-cavity surface-emitting lasers (VCSELs) and photodiodes (PDs) with micron-scale accuracy. An important factor that causes EMI radiation in optical modules and systems, and the transmission lines and interconnect structure of the transmitter and receiver boards were carefully designed for reducing EMI noise. In this system, a $4.5 \mathrm{~Gb} / \mathrm{s}$ data transmission rate demonstrated between the Tx chips and the $R x$ chips without EMI problems. The eye was wide open and showed no relaxation oscillation.

\section{REFERENCES}

[1] E. Griese, "Reducing EMC problems through an electrical/optical in-terconnection technology," IEEE Trans. Electromagn. Compat., vol. 41, no. 4, pp. 502-509, Nov. 1999

[2] S. H. Hwang et al., "Passively assembled optical interconnection system based on an optical printed-circuit board,"IEEE Photon. Technol. Lett., vol. 18, no. 5, pp. 652-654, Mar. 2006.

[3] Y.-T. Han, J.-U. Shin, S.-H. Park, S.-P. Han, C.-H. Lee, Y.-O. Noh, H.-J. Lee, and Y.-S. Baek,
"Crosstalk-enhanced DOS integrate with modified radiation-type attenuators," ETRI J., vol. 30, no. 5, pp. 744-746, 2008.

[4] H. S. Cho, K.-M. Chu, S. K. Kang, and H.-H. Park, "Compact packaging of optical and electronic components for on-board optical interconnects," IEEE Trans. Adv. Packag., vol. 28, no. 1, pp. 114-120, Feb.2005.

[5] I.-K. Choet al., "Experimental demonstration of 10 Gbit/s transmission with an optical backplane system using optical slots," Opt. Lett., vol. 30, no. 13, pp. 1635-1637, 2005.

[6] E. Grivas et al., "Wavelength division multiplexing based optical backplane with arrayed waveguide grating passive router," Opt. Eng., vol. 47, pp. 025401-, 2008.

[7] R. Dangel, C. Berger, R. Beyeler, and T. Morf, "Polymer-waveguide-based board-level optical interconnect technology for datacom applications," IEEE Trans. Adv. Packag., vol. 31, no. 4, pp. 759-767, Nov.2008.

[8] R. Bockstaele et al., "Realization and characterization of 828 resonant cavity LED arrays mounted onto CMOS drivers for POF-based interchip interconnections," IEEE J. Sel. Topics Quantum Electron., vol.5, pp. 224-235, Sep./Oct. 1999.

[9] B. Wittmann, M. Jöhnck, A. Neyer, F. Mederer, R. King, and R. Michalzik, "POF-based interconnects for intracomputer applications," IEEE J. Sel. Topics Quantum Electron., vol. 5, pp. 1243-1248, Sep./Oct. 1999.

[10] V. Ozguz, "Chip-level three-dimensional stacking for free-space optical interconnects," in Proc. Conf. Lasers Electro-Optics (CLEO),1998, pp. 352-353.

[11 I.-K. Cho et al., "Optical module using polymer waveguide with integrated reflector mirrors," IEEE Photon. Technol. Lett., vol. 20, no. 6,pp. 410-412, Mar. 2008. 